EPJ Web of Conferences 71, 00062 (2014)

DOI: 10.1051/epjconf/20147100062

(C) Owned by the authors, published by EDP Sciences, 2014

\title{
Ultra-high energy physics and standard basic principles
}

\section{Do Planck units really make sense?}

\author{
Luis Gonzalez-Mestres ${ }^{1, a}$ \\ ${ }^{1}$ Megatrend Cosmology Laboratory, Megatrend University, Belgrade and Paris \\ Goce Delceva 8, 11070 Novi Beograd, Serbia
}

\begin{abstract}
It has not yet been elucidated whether the observed flux suppression for ultrahigh energy cosmic rays (UHECR) at energies above $\simeq 4 \times 10^{19} \mathrm{eV}$ is a signature of the Greisen-Zatsepin-Kuzmin (GZK) cutoff or a consequence of other phenomena. In both cases, violations of the standard fundamental principles of Physics can be present and play a significant role. They can in particular modify cosmic-ray interactions, propagation or acceleration at very high energy. Thus, in a long-term program, UHECR data can hopefully be used to test relativity, quantum mechanics, energy and momentum conservation, vacuum properties... as well as the elementariness of standard particles. Data on cosmic rays at energies $\simeq 10^{20} \mathrm{eV}$ may also be sensitive to new physics generated well beyond Planck scale. A typical example is provided by the search for possible signatures of a Lorentz symmetry violation (LSV) associated to a privileged local reference frame (the "vacuum rest frame", VRF). If a VRF exists, the internal structure of standard particles at ultra-high energy can undergo substantial modifications. Similarly, the conventional particle symmetries may cease to be valid at such energies instead of heading to a grand unification and the structure of vacuum may no longer be governed by standard quantum field theory. Then, the question whether the notion of Planck scale still makes sense clearly becomes relevant and the very grounds of Cosmology can undergo essential modifications. UHECR studies naturally interact with the interpretation of WMAP and Planck observations. Recent Planck data analyses tend to confirm the possible existence of a privileged space direction. If the observed phenomenon turns out to be a signature of the spinorial space-time (SST) we suggested in 1996-97, then conventional Particle Physics may correspond to the local properties of standard matter at low enough energy and large enough distances. This would clearly strengthen the cosmological relevance of UHECR phenomenology and weaken the status of the Planck scale hypothesis. Another crucial observation is that, already before incorporating standard matter and relativity, the SST geometry naturally yields a $H t=1$ law where $t$ is the age of the Universe and $H$ the ratio between relative speeds and distances at cosmic scale. As standard cosmology is not required to get such a fundamental result, the need for a conventional Planck scale is far from obvious and the study of UHECR can potentially yield evidence for an alternative approach including new physics and new ultimate constituents of matter. UHECR may in particular allow to explore the possible indications of the existence of a transition scale at very high energy where the standard laws would start becoming less and less dominant and new physics would replace the conventional fundamental principles. We discuss prospects of searches for potential signatures of such a phenomenon.
\end{abstract}

ae-mail: luis.gonzalez-mestres@cosmology.megatrend.edu.rs 


\section{Introduction}

Recent measurements and analyses [1,2] have not yet allowed to elucidate whether the observed UHECR flux suppression above $\simeq 4 \times 10^{19} \mathrm{eV}[3,4]$ is really a signature of the Greisen-ZatsepinKuzmin (GZK) cutoff $[5,6]$ or corresponds to a flux-suppressing mechanism of a different dynamical or astrophysical origin. All possible situations can be sensitive to new physics modifying at very high energy the production of cosmic rays $[7,8]$, their propagation $[9,10]$ or their acceleration at astrophysical sources $[11,12]$. Various violations of the standard basic principles of current particle physics (relativity, quantum mechanics and quantum field theory (QFT), energy and momentum conservation, vacuum homogeneity and "static" properties, effective space dimensions, symmetries, quark confinement, conventional elementariness of particles...) can be potentially involved $([13,14]$ and $[15,16])$. New physics may even be present in data above $\simeq 10^{16} \mathrm{eV}[17,18]$. Data from UHECR experiments are in particular expected $[9,12]$ to yield bounds on Lorentz symmetry violation (LSV) parameters in models incorporating a privileged local reference frame (the "vacuum rest frame", VRF $[19,20])$. But the phenomenological analysis of experimental data at ultra-high energy (UHE) will be a difficult task and requires a long-term experimental and theoretical effort [14, 21] having in mind all possible forms of LSV as well potential violations of other fundamental principles of standard physics and the necessary compatibility with low-energy bounds on LSV [22, 23]. It can be readily checked, for instance, that LSV violations leading to spontaneous decays of UHECR may fake the GZK cutoff $[24,25]$. A typical example was provided in [21], where the GZK cutoff would be replaced by spontaneous emission of photons or $\mathrm{e}^{+} \mathrm{e}^{-}$pairs in a simple LSV pattern.

The Planck scale is often taken as a reference to parameterize possible deviations from standard physics at very high energy and at very small distances. But this choice does not necessarily make sense $[13,14]$, as new physics may become dominant at energies below Planck energy and at distances above the Planck distance scale [21, 26, 27]. Contrary to approaches to quantum gravity [28, 29] where the role of LSV is basically reduced to deformations and extensions of existing algebras [30, 31], the approach based on the existence of a local VRF for each comoving observer explicitly breaks the associated symmetries that become low-energy limits just as the Lorentz-like symmetry of the kinematics of phonons in a lattice [9, 19]. Then, extrapolations from low-energy physics such as Grand Unification and the standard concept of gravitation do not necessarily remain valid at very high energy and very small distances. Similarly, the speed of light $c$ may cease to dominate the natural relation between energy and momentum or between distance and time scales, and the Planck constant $h$ may no longer be relevant to relate energy and time scales. In this case, two new scales of energy, momentum, distance and time become relevant: i) the fundamental scale ( $E_{a}$ in terms of energy) at which a new physics is generated; ii) the transition scale $E$ (energy) $\approx E_{\text {trans }}$ at which the new physics starts becoming more and more significant in experimental data. New ultimate constituents of matter (e.g. superbradyons, superluminal preons) can also be present in such scenarios [19, 32] where standard internal symmetries may cease to make sense at high enough energy $[15,16]$ instead of becoming more exact as energy increases. Superbradyons can be part of the dark matter [8, 15].

Simultaneously, cosmological studies are expected to cast light on the same basic questions, in particular through WMAP [33] and Planck [34] data [35, 36] including the possible signature of a privileged space direction as the Planck analysis of maps of the cosmic microwave background (CMB) anisotropy [37] seems to suggest. The existence of such a privileged space direction for each comoving observer is a natural prediction of the spinorial space-time (SST) geometry we suggested in 1996-97 [8, 38]. No standard matter or conventional relativity is required to get such a result. 
Thus, the SST pattern combined with parity violation can potentially account for Planck and WMAP observations $[39,40]$. Similarly, previous to any introduction of conventional matter and relativity, the SST leads by itself in an automatic way $[12,13]$ to the relation $H t=1$ where $t$ is the cosmic time (age of the Universe) and $H$ the Lundmark-Lemaitre-Hubble (LLH) constant describing the ratio between relative velocities and distances at cosmic scales. The $H t=1$ law thus emerges as a natural asymptotic limit of the evolution of the Universe in a SST cosmic geometry [41, 42]. The fact that such results can be obtained without using standard physics clearly deserves a closer analysis [13, 41]. In particular, the conventional principles of Physics obtained from particle experiments may happen to be valid only as a low-energy limit in the regions of the Universe where standard matter has nucleated. Such a scenario would confirm the picture already suggested in $[9,19]$ and in $[8,43]$. In particular, if a VRF associated to LSV exists, high-energy particles are obviously not the same objects as particles at rest. LSV and other new physics can then modify the internal structure, symmetries and interaction properties of particles at very high energy. Such a new physics can possibly be related to pre-Big Bang scenarios $[13,41]$ and to new ultimate constituents of matter. Thus, Cosmology and UHECR studies should permanently interact in a common attempt to search for new signatures of new phenomena and hopefully understand the ultimate properties of matter, possible pre-matter and space-time.

In a pre-Big Bang approach with the spinorial space-time, if standard matter has nucleated on a pre-existing vacuum associated to the SST geometry, new cosmological forces can be generated by this transition. In particular, the gravitationally coupled matter can react to the pre-existing LLH expansion of the Universe and generate a new form of energy and cosmological force and pressure [42]. This reaction would become weaker as the density of the standard matter decreases with the Universe expansion and the relation $H t=1$ would remain a suitable asymptotic limit at large $t$.

Another essential question is that of the role of high-energy accelerator experiments. Signatures of supersymmetry do not seem to have been found by now at LHC, and our analysis [45, 46] of the September 2011 OPERA claim [44] on the possible detection of a superluminal neutrino pointed out that such a comparatively strong effect could not be made consistent with existing and well-established cosmic-ray and astrophysical data. Even assuming that supersymmetry exists, the whole pattern can fail at higher energies it Lorentz symmetry is violated and a VRF exists. But finding a measurable signature of LSV at present accelerators can be a very hard task. The possibility to search for decays producing preon-like objects (superbradyons...), assuming they can exist as free particles in our Universe and be produced at accelerators, was considered in [15, 47]. Astrophysical observations can also be relevant, as cosmic superbradyons are expected to spontaneously emit "Cherenkow" radiation in vacuum in the form of standard particles when moving at a speed larger than $c$ or close to this value $[12,19]$. Superbradyon decays can even be a source of high-energy cosmic rays $[7,8]$.

We present here and updated discussion of the relevance of UHECR data for fundamental Particle Physics and Cosmology, emphasizing specific opportunities related to possible new physics and preBig Bang scenarios. The question of the relevance of the Planck scale is explicitly dealt with.

\section{An illustrative example: LSV kinematics}

Crucial for the possible existence of specific signatures of new physics in UHECR data is Lorentz symmetry violation with a vacuum rest frame. The Earth is then assumed to move slowly with respect to the local VRF. Then, the observable properties of UHECR can reflect new physics generated at a new fundamental scale $E_{a}$, not only concerning space-time properties but more generally including the fate of particle symmetries and interactions or the properties of matter and pre-matter.

LSV patterns for kinematics can be used to illustrate possible descriptions of this new physics. Ways to deal with possible violations of standard quantum mechanics at high energy have been considered in $[14,48]$. In most cases, the smallness of the mass term of a UHECR turns out to be a crucial 
property to make the UHECR properties and interactions sensitive enough to very small effects from unconventional physics impossible to observe with lower-energy particles.

\subsection{A simple model and some estimates}

An illustrative example, using LSV with a VRF, can be provided by the quadratically deformed relativistic kinematics (QDRK) defined by the relation $[9,10]$ :

$$
E \simeq p c+m^{2} c^{3}(2 p)^{-1}-p c \alpha\left(p c E_{a}^{-1}\right)^{2} / 2
$$

where $p$ is the momentum assumed to be $\ll E_{a} c^{-1}, m$ the mass, $\alpha$ a positive constant describing the strength of the deformation and $E_{a}$ the effective fundamental energy scale. Then, the negative deformation term:

$$
\Delta E \simeq-p c \alpha\left(p c E_{a}^{-1}\right)^{2} / 2
$$

becomes larger than the positive mass term $m^{2} c^{3}(2 p)^{-1}$ above a transition energy $E_{\text {trans }}$ :

$$
E_{\text {trans }} \simeq \alpha^{-1 / 4}\left(E_{a} m\right)^{1 / 2} c
$$

It immediately follows that for a proton at ultra-high energy (UHE) with $\alpha=1$, one would have $E_{\text {trans }} \simeq 10^{20} \mathrm{eV}$ if $E_{a} \simeq 10^{3} E_{\text {Planck }}$ where $E_{\text {Planck }}$ is the Planck energy. Therefore, a UHE proton would be potentially sensitive to new physics generated three orders of magnitude beyond the Planck energy scale [14, 49]. Other possible values of the LSV parameters have been considered in $[12,25]$.

Furthermore, writing:

$$
\begin{gathered}
\Delta E \simeq-\alpha E\left(E E_{a}^{-1}\right)^{2} / 2 \\
E_{m} \simeq E_{\text {rest }}^{2} E^{-1} / 2
\end{gathered}
$$

where $E_{m}$ is the kinematical mass term and $E_{\text {rest }}=m c^{2}$ the rest energy of the particle, and taking $\alpha=1$, the relation

$$
\Delta E E_{m}^{-1} \simeq-E^{4}\left(E_{a} E_{\text {rest }}\right)^{-2}
$$

makes explicit the naturally strong energy dependence of the deformation at very high momentum.

The sharp rise of the ratio $\Delta E E_{m}^{-1}$ thus obtained from QDRK can be the sign of a strong transition for $E>E_{\text {trans }}$ if the mass term $E_{m}$ is interpreted as an expression of the original internal structure of the particle considered. At the Grand Unification scale $E \simeq 10^{16} \mathrm{GeV}$, one would have $\Delta E E_{m}^{-1} \approx 10^{20}$ for a proton with $\alpha=1$ and $E_{a} \simeq 10^{3} E_{\text {Planck. }}$. At the Planck energy scale, $\Delta E E_{m}^{-1} \approx 10^{32}$ and $\Delta E E^{-1} \approx 10^{-6}$.

Assuming QDRK to be correct, such very large values of $\Delta E E_{m}^{-1}$ would clearly suggest a possible change in the UHECR internal structure and dynamics at energies above $E_{\text {trans }}$ and already well below the grand unified and Planck energy scales.

Another possible parameterization, equivalent to QDRK, can be:

$$
E^{2}=p^{2} c^{2}+m^{2} c^{4}-(\delta E)^{2}
$$

where $\delta E$ is assumed to vary proportionally to $\simeq E^{2}$ at high energy.

Then, if $\delta E=m c^{2}$ (defining the transition energy) at $E \simeq 10^{20} \mathrm{eV}$, one has $\delta E E_{\text {rest }}^{-1} \simeq 10^{10}$ at $E \simeq 10^{16} \mathrm{GeV}$ and $\delta E E_{\text {rest }}^{-1} \simeq 10^{16}$ at the Planck energy scale.

Approaches based on and (1) or (7) have also implications on the motion of the particle. Above $E_{\text {trans }}$, the Hamiltonian velocity $v=d E / d p$ does no longer tend to $c$ as the energy increases. Instead, the positive difference $c-v$ increases with energy. Similarly, the ratio $E p^{-1}$ becomes smaller than 
$c$ above $E_{\text {trans }}$. This is substantially different from the standard relativistic scenario where the relative difference between $c$ and $v$ becomes as small as $\approx 10^{-38}$ for a proton at the Planck energy scale. Instead, one would get from (1):

$$
d E / d p \simeq c-m^{2} c^{3}\left(2 p^{2}\right)^{-1}-3 c \alpha\left(p c E_{a}^{-1}\right)^{2} / 2
$$

leading, for a proton with $E_{\text {trans }} \simeq 10^{20} \mathrm{eV}$, to $c-d E / d p \simeq 10^{-6} c$ at the Planck energy scale.

\subsection{Theoretical and phenomenological considerations}

A natural alternative to QDRK would be to replace the deformation term (2) by an expression depending quadratically on $p$. But we rejected [43] this linearly deformed relativistic kinematics (LDRK) because of its too strong predictions at low energy as compared to experimental bounds [22, 23].

The value of $\alpha$ in the QDRK equation (1) is actually not expected to be necessarily the same for all standard particles $[12,25]$. Similarly, the universality of $E_{a}$ appears as a natural choice but the real dynamics can be more complex. Here, $\alpha$ and $E_{a}$ refer basically to effective values for each particle considered. Although Grand Unification may suggest taking the same values $\alpha$ and $E_{a}$ for all quarks and leptons, such a symmetry is not necessarily relevant at the fundamental energy scale where new physics can play a leading role. Contrary to conventional prejudices, low-energy symmetries can actually be a way to hide essential properties of the structure of standard particles at very high energy and very short distances where a new kind of matter or pre-matter possibly dominates.

According to conventional quantum mechanics and relativity, $\mathrm{a} \approx 10^{20} \mathrm{eV}$ energy scale corresponds to $\mathrm{a} \approx 10^{-25} \mathrm{~cm}$ distance scale, 12 orders of magnitude below the proton radius $(\simeq 0.9 \mathrm{x}$ $10^{-13} \mathrm{~cm}$ with electric dipole moment $\simeq 5.4 \times 10^{-24} e . \mathrm{cm}$ where $e$ is the elementary charge). Lower values of $E_{\text {trans }}$ are not excluded by data $[12,25]$, in particular if the standard GZK cutoff is not the mechanism at work above $\simeq 4 \times 10^{19} \mathrm{eV}$. For a proton, $E_{\text {trans }} \simeq 3 \times 10^{19} \mathrm{eV}$, just below the observed fall of the spectrum, would be obtained with $E_{a} \simeq 100 E_{\text {Planck }}$ and $\alpha=1$. Taking $E_{a} \simeq 10 E_{\text {Planck }}$ and $\alpha=1$ yields $E_{\text {trans }} \simeq 10^{19} \mathrm{eV}$. In this case, one would have : i) $\Delta E E_{m}^{-1} \approx 10^{24}$ and $\delta E E_{\text {rest }}^{-1} \simeq 10^{12}$ at the Grand Unification scale $E \simeq 10^{16} \mathrm{GeV}$; ii) $\Delta E E_{m}^{-1} \approx 10^{36}, \Delta E E^{-1} \approx 10^{-4}$ and $\delta E E_{\text {rest }}^{-1} \simeq 10^{18}\left(\delta E \simeq 0.1 E_{\text {Planck }}\right)$ at the Planck energy scale.

For an electron, using (1) and (3) with $\alpha=1$ and $E_{a} \simeq 10^{3} E_{\text {Planck }}$ yields $E_{\text {trans }} \simeq 2 \times 10^{18}$ $\mathrm{eV}$. Taking instead $\alpha=10^{-2}$ would lead to $E_{\text {trans }} \simeq 2 \times 10^{19} \mathrm{eV}$ corresponding to a $\approx 10^{-24} \mathrm{~cm}$ distance scale. The ACME bound [50] on the electric dipole moment of the electron is $\left|d_{e}\right|<8.7 \mathrm{x}$ $10^{-29} e \mathrm{~cm}$, where $d_{e}$ is the dipole moment. For a muon with $\alpha \simeq 1$ and $E_{a} \simeq 10^{3} E_{\text {Planck }}$, one gets $E_{\text {trans }} \simeq 3 \times 10^{19} \mathrm{eV}$. A bound on the muon electric dipole moment is [51] $\left|d_{\mu}\right|<1.9 \times 10^{-19} e \mathrm{~cm}$, where $d_{\mu}$ is the muon electric dipole moment.

If a nucleus is dealt with to a first approximation as an additive set of $N$ nucleons, the associated effective value of $E_{\text {trans }}$ becomes $N$ times larger than for protons [12, 25]. This does not appears to be inconsistent with AUGER measurements [1,3]. The detailed study of UHECR composition is a crucial ingredient of the test of basic principles through AUGER data. Another possibility to be considered is quark deconfinement at ultra-high energy, especially given the fact that the parton model cannot work at UHE [52, 53]. A cosmic free quark can satisfy equation (1) with an effective value of $\alpha$ well above that associated to the proton $[12,25]$. This property may help deconfinement. If $E_{Q f r e e}$ is the minimum energy of a free quark, a quark with energy $E>E_{Q \text { free }}$ can release an energy $\simeq E-E_{Q f \text { ree }}$ in the atmosphere. After having released most of this energy, it can still produce significant effects through hadron mutations. For instance, a quark (antiquark) with charge $2 / 3(-1 / 3)$ can replace a quark with charge - 1/3 (2/3) inside a hadron, turning a neutron (proton) into a proton (neutron). The considered deconfinement at UHE is in principle a phase transition and the sequential 
absorption of nucleons by free quarks considered in [54] would not necessarily be possible in the present approach, as the quark would itself become much slower when absorbing hadrons and nuclei.

Other possible UHE effects and tests of LSV have been discussed in [12, 14]. In the presence of LSV with a VRF, additional violations of standard basic principles can also produce observable signatures at ultra-high energy [13,14]. It must be noticed that (1) and (2) include a possible deformation of the effective value of the speed of light through the $\Delta E$ term. For a photon with $\alpha=1$ , $E_{a} \simeq 10^{3} E_{\text {Planck }}$ and $E=10^{19} \mathrm{eV}$, one would have $E p^{-1}-c \simeq-3 \times 10^{-25} c$ and $d E / d p-c \simeq-10^{-24} c$. These values are much smaller than those considered at lower energies in connection with the OPERA claim of september 2011 [44]. On the other hand, the photon internal structure can be significantly modified by this apparently small deviation from the standard critical speed $c$. Similarly, specific models describing violations of other fundamental principles (quantum mechanics...) will also tacitly include an effective energy dependence of other fundamental constants (the Planck constant $h$...). Coupling constants and interaction properties can undergo a stronger evolution as energy increases, due to the role of effective masses and particle internal structure. As a consequence, the validity of cosmic-ray energy estimations may become uncertain at UHE.

From a cosmological point of view, the values $E \simeq 10^{19} \mathrm{eV}$ and $E \simeq 10^{20} \mathrm{eV}$ correspond respectively to temperatures $T \simeq 10^{23} \mathrm{~K}$ and $T \simeq 10^{24} \mathrm{~K}$ in the history of the Universe. At a temperature scale of $T \simeq 10^{27} \mathrm{~K}$ or $T \simeq 10^{28} \mathrm{~K}$ usually associated to inflation, the ratios $\Delta E E_{m}^{-1}$ and $\delta E E_{\text {rest }}^{-1}$ would already have large values. Then, the evolution of a pre-Big Bang scenario can reasonably replace the standard Big Bang and inflation. In pre-Big Bang patterns, the formation of standard matter can just correspond to a phase transition or be associated to a long evolution of the properties of matter and pre-matter. UHECR phenomenology is directly concerned by such fundamental questions and should be used to search for relevant cosmological signatures, including tracks of possible free UHE preonic objects such as superbradyons [7, 32].

In connection with UHE physics and cosmological observations of the early Universe [13, 41], the internal structure of particles at very small distances is also a relevant subject including possible preonic (superbradyonic?) structures. The SST geometry, that may have successfully predicted [12, 38-40] the existence of a privileged space direction as observed by Planck [37], can hopefully be used to describe both the structure and evolution of the Universe at very large scale and the particle structure and dynamics at very small distances $[12,41]$. In the SST, the space-time position spinor $\xi$ is made of two complex coordinates transforming under a $\mathrm{SU}(2)$ group associated to space rotations $[13,38]$. The cosmic time $t$ (age of the Universe) is given by the spinor modulus $|\xi|$ or a function of this modulus. Taking $t=|\xi|$, the $S^{3}$ hypersphere formed by all spinors of modulus $t$ defines the space at time $t$. If $\xi_{0}$ is the spinorial position of the observer at time $t$, the spatial coordinates of a point $\xi$ on the same constant-time hypersphere are defined by the path from $\xi_{0}$ to $\xi$ on the hypersphere. A position vector can thus be readily built using the relevant $S U(2)$ transformation $[38,41]$. However, it is also possible $[12,39]$ to consider spinorial local coordinates if the requirement of constant cosmic time for the path between $\xi_{0}$ and $\xi$ is abandoned. The difference $\Delta \xi=\xi-\xi_{0}$, which changes sign under a $2 \pi \mathrm{SU}(2)$ rotation, provides a relative position spinor of $\xi$ with respect to $\xi_{0}$. The spinorial coordinates can be relevant at very small distances to describe the internal structure of a fermion in terms of its ultimate constituents. Trying to find a track of this spinorial internal structure in data on UHECR would be an important theoretical, experimental and phenomenological challenge.

While many questions remain open, the ratios $\Delta E E_{m}^{-1}$ and $\delta E E_{r e s t}^{-1}$ obtained for $E_{a} \simeq 10^{20} \mathrm{eV}$ and $E_{a} \simeq 10^{19} \mathrm{eV}$ clearly suggest that the internal structure of standard particles, as well as their interaction properties, can considerably evolve between low energy and the Grand Unification scale or the Planck energy scale, and strongly deviate from conventional patterns above $E_{\text {trans }}$. Quantum mechanics itself can be significantly modified or even cease to be valid. In such a situation, the 
conventional Grand Unified and Planck scales do not necessarily make sense. Similarly, the idea that low-energy symmetries should become more and more exact as energy increases has no reason to hold above $E_{\text {trans }}[12,21]$. Precise particle identification can therefore be an important ingredient of UHECR experiments and phenomenology. Another difficult task will be to take into account possible deviations from standard particle dynamics when analyzing UHECR interactions with the atmosphere.

\section{UHECR internal structure}

Equations (1) and (2) were obtained $[9,10]$ from an analogy with the solid state Bravais lattice writing the standard equation for phonon kinematics:

$$
d^{2} / d t^{2}[\phi(n)]=-K[2 \phi(n)-\phi(n-1)-\phi(n+1)]-\omega_{\text {rest }}^{2} \phi(n)
$$

where $n$ (integer) stands for the site under consideration, $\phi(n)$ is a complex order parameter, $K$ an elastic constant and $\omega_{\text {rest }}$ the frequency of the chain of oscillators in the zero-momentum limit. Then:

$$
\omega^{2}(k)=2 K[1-\cos (k a)]+\omega_{r e s t}^{2}=4 K \sin ^{2}(k a / 2)+\omega_{r e s t}^{2}
$$

$\omega$ being the frequency, $k$ the wave vector and $a$ the lattice spacing. QDRK is similar to the power expansion of (10) in terms of $k a$ for $k a \ll 1$ replacing the speed of sound by the speed of light and the lattice spacing by the fundamental length scale.

Another analogy was already introduced in our 1995 papers [19, 20] comparing standard elementary particles to condensed-matter solitons. Then, the critical speed of the ultimate constituents of matter can be much larger than that of light, just as the speed of light is much larger than that of sound. The soliton analogy was further explored in [52, 53]. Similar to this analysis, one can consider the equation:

$$
c^{-2} \partial^{2} \Psi / \partial t^{2}-\partial^{2} \Psi / \partial x^{2}=2 \Delta^{-2} \Psi(1-2 \Psi)
$$

$\Psi$ being a real function of $x$ and $t(x=$ space coordinate, $t=$ time coordinate) and $\Delta$ the distance scale that characterizes the soliton size at rest (soliton radius). The soliton solution to equation (11) is:

$$
\Psi(x, t)=1-\tanh ^{2}\left(\lambda_{0} y\right)
$$

where $y=x-v t, v$ is the speed of the soliton, $\lambda_{0}=\Delta^{-1} \gamma_{R}, D$ is the rest radius of the soliton and $\gamma_{R}$ the standard relativistic Lorentz factor $\gamma_{R}=\left(1-v^{2} c^{-2}\right)^{-1 / 2}$. As conventional Lorentz contraction and time dilation are altered by LSV, this analogy allows in particular to explore the possible energy dependence of the effective particle size above the relevant transition energy.

Adding to equation (11) a term proportional to $a^{2} \partial^{4} \Psi / \partial x^{4}$ ( $a=$ fundamental length) can be considered as the equivalent of (1) and indeed leads to a similar kinematics [52, 53].

A simplified discussion of the effect of such a deformation of equations (11) and (12) can be as follows. Applying the operator $a^{2} \partial^{4} / \partial x^{4}$ to the initial solution given by (12) yields a result proportional to $a^{2} \gamma_{R}^{4} D^{-4}$ that becomes much larger than $D^{-2}$ for $a \gamma_{R}^{2} \gg D$. A substantial modification of (11) and (12) is then required reproducing the maximum speed lower than $c$ of QDRK. If the existence of a soliton can be preserved at UHE, the new equation should admit two branches of solutions (above and below the energy associated to the maximum of $v$ ). LSV will become dominant at ultra-high energy and introduce a contraction law different from the relativistic one. This result suggests once more the possible existence of a sharp transition in the UHECR internal structure and interaction properties.

For a proton with $E \simeq 10^{20} \mathrm{eV}\left(\gamma_{R} \simeq 10^{11}\right)$ and $a \simeq 10^{-36} \mathrm{~cm}\left(\simeq 10^{-3} l_{\text {Planck }}, l_{\text {Planck }}=\right.$ Planck length), one would have $a \gamma_{R}^{2} \simeq 10^{-14} \mathrm{~cm}$ which is already significant as compared to the rest proton radius. Taking instead $a=0.1 l_{\text {Planck }}$ would lead at $E \simeq 10^{20} \mathrm{eV}$ to $a \gamma_{R}^{2} \simeq 10^{-12} \mathrm{~cm}$ that is larger than the rest proton radius. Therefore, the deformation of Lorentz contraction just considered can already be present in UHECR and produce observable signatures. 


\section{Conclusion}

The highest-energy cosmic rays are a crucial experimental source of fundamental information for Particle Physics and Cosmology. They require careful exploration and study within a long-term program of Earth-based and satellite experiments, with particular attention to the first interactions of the incoming cosmic rays. If unconventional new physics exists, the fate of concepts such as the standard Planck scale and the grand unified scale appears very uncertain. UHECR experiments can then provide a unique way to help particle theory and cosmological research to uncover the actual structure and origin of matter and space-time, including the search for a possible correlation between the arrival directions of UHECR and the SST privileged space direction that Planck data may have disclosed.

Observable LSV signatures may come not only from deformations of kinematics $[9,10]$ but also from significant changes in the UHE particle internal structure $[52,53]$ expected to modify the interaction properties for nucleons, leptons and photons. But elucidating the nature of the actual dynamics that generates the observed data (including a possible absence of deviations from standard physics) will be a very difficult, long-term task. In parallel, the study of potential UHECR sources should be pursued as far as possible without forgetting nonstandard mechanisms (superbradyon decays [7, 8]...).

\section{References}

[1] A. Letessier-Selvon for the Pierre Auger Collaboration, Highlights from the Pierre Auger Observatory, 33rd International Cosmic Ray Conference (ICRC 2013), Rio de Janeiro 2013.

[2] J.R.T. de Mello Neto for the Pierre Auger Collaboration, Measurements of cosmic rays at the highest energies with the Pierre Auger Observatory, arXiv:1309.1249

[3] The Pierre Auger Observatory: Contributions to the 33rd International Cosmic Ray Conference (ICRC 2013).

[4] T. Abu-Zayyad et al., Energy Spectrum of Ultra-High Energy Cosmic Rays Observed with the Telescope Array Using a Hybrid Technique, arXiv:1305.7273

[5] K. Greisen, End to the Cosmic-Ray Spectrum? Phys.Rev.Lett. 16 (1966), 748.

[6] G.T. Zatsepin and V.A. Kuz'min, Upper Limit on the Spectrum of Cosmic Rays, JETP Letters 4, 78

[7] L. Gonzalez-Mestres, Superluminal Matter and High-Energy Cosmic Rays, arXiv:astro$\mathrm{ph} / 9606054$

[8] L. Gonzalez-Mestres, Physical and Cosmological Implications of a Possible Class of Particles Able to Travel Faster than Light, contribution to the 28th International Conference on High Energy Physics, Warsaw 1996, arXiv:hep-ph/9610474, and references therein.

[9] L. Gonzalez-Mestres, Vacuum Structure, Lorentz Symmetry and Superluminal Particles, arXiv:physics/9704017

[10] L. Gonzalez-Mestres, Absence of Greisen-Zatsepin-Kuzmin Cutoff and Stability of Unstable Particles at Very High Energy, as a consequence of Lorentz Symmetry Violation, Proceedings of the 25th International Cosmic Ray Conference, Potchefstroomse Universiteit 1997, Vol. 6, p. 113, SAO/NASA record.

[11] L. Gonzalez-Mestres, Lorentz Symmetry Violation and Acceleration in Relativistic Shocks, Proceedings of the Heidelberg 2000 International Symposium on High-Energy $\gamma$-Ray Astrophysics, AIP Conf.Proc. 558 (2001), 874, arXiv:astro-ph/0011182.

[12] L. Gonzalez-Mestres, Cosmic rays and tests of fundamental principles, CRIS 2010 Proceedings, Nucl. Phys. B, Proc. Suppl. 212-213 (2011), 26, and references therein. The arXiv.org version arXiv:1011.4889 includes a relevant Post Scriptum. 
[13] L. Gonzalez-Mestres, Pre-Big Bang, fundamental Physics and noncyclic cosmologies, presented at the International Conference on New Frontiers in Physics, ICFP 2012, Kolymbari, Crete, June 10-16 2012, mp_arc 13-18, and references therein.

[14] L. Gonzalez-Mestres, High-energy cosmic rays and tests of basic principles of Physics, presented at the International Conference on New Frontiers in Physics, ICFP 2012, Kolymbari, Crete, June 10-16 2012, mp_arc 13-19, and references therein.

[15] L. Gonzalez-Mestres, Superbradyons and some possible dark matter signatures, arXiv:0905.4146

[16] L. Gonzalez-Mestres, Lorentz symmetry violation, dark matter and dark energy, Invisible Universe International Conference, Paris 2009, AIP Conf.Proc. 1241 (2010), 1207. The arXiv.org version arXiv:0912.0725 contains a relevant Post Scriptum.

[17] I.P. Lokhtin, A.K. Managadze and A.M. Snigirev, Ridge effect and alignment phenomenon, Physics of Atomic Nuclei 76 (2013), 602.

[18] L. Gonzalez-Mestres, Lorentz violation, vacuum, cosmic rays, superbradyons and Pamir data, arXiv:astro-ph/1009.1853

[19] L. Gonzalez-Mestres, Properties of a possible class of particles able to travel faster than light, Proceedings of the January 1995 Moriond Workshop, Ed. Frontières, arXiv:astro-ph/9505117

[20] L. Gonzalez-Mestres, Cosmological Implications of a Possible Class of Particles Able to Travel Faster than Light Proceedings of the TAUP 1995 Conference, Nucl. Phys. Proc. Suppl. 48 (1996), 131, arXiv.org, arXiv:astro-ph/9601090

[21] L. Gonzalez-Mestres, Testing fundamental principles with high-energy cosmic rays, 2011 Europhysics Conference on High Energy Physics, Grenoble, July 2011, PoS EPS-HEP2011 (2011) 390 , and references therein.

[22] See, for instance, S.K. Lamoreaux, Relativity: Testing times in space, Nature 416 (2002), 803, and references therein.

[23] For more recent measurements, see for instance M.A. Hohensee et al., Limits on Violations of Lorentz Symmetry and the Einstein Equivalence Principle using Radio-Frequency Spectroscopy of Atomic Dysprosium, Phys. Rev. Lett. 111 (2013), 050401, arXiv:1303.2747

[24] L. Gonzalez-Mestres, Lorentz symmetry violation and the results of the AUGER experiment, arXiv:0802.2536

[25] L. Gonzalez-Mestres, AUGER-HiRes results and models of Lorentz symmetry violation, CRIS 2008 Proceedings, Nucl. Phys. Proc. Suppl. 190 (2009), 191, arXiv:0902.0994, and references therein.

[26] L. Gonzalez-Mestres, Pre-Big Bang, vacuum and noncyclic cosmologies, 2011 Europhysics Conference on High Energy Physics, PoS EPS-HEP2011(2011) 479, and references therein.

[27] L. Gonzalez-Mestres, WMAP, Planck, cosmic rays and unconventional cosmologies, contribution to the Planck 2011 Conference, Paris, January 2011, arXiv:1110.6171.

[28] C. Kiefer, Conceptual Problems in Quantum Gravity and Quantum Cosmology, ISRN Math.Phys. 2013 (2013), 509316, arXiv:1401.3578

[29] A. Ashtekar, Loop Quantum Gravity and the The Planck Regime of Cosmology, arXiv:1303.4989

[30] G. Amelino-Camelia et al., Relative locality: A deepening of the relativity principle, arXiv:1106.0313

[31] L. Smolin, General relativity as the equation of state of spin foam, arXiv: 1205.5529

[32] L. Gonzalez-Mestres, Observing air showers from cosmic superluminal particles, Workshop on "Observing Giant Cosmic Ray Air Showers for > 10E20 eV Particles from Space", University of Maryland, November 1997, AIP Conf. Proc. 433, 418 (1998), arXiv:physics/9712049 
[33] Wilkinson Microwave Anisotropy Probe (WMAP), http://map.gsfc.nasa.gov/

[34] Planck mission (European State Agency, ESA), http://sci.esa.int/sciencee/www/area/index.cfm?fareaid $=17$

[35] Planck Collaboration, Planck 2013 results. I. Overview of products and scientific results, arXiv: 1303.5062

[36] Planck Collaboration, Planck 2013 results. XVI. Cosmological parameters, arXiv:1303.5076

[37] The Planck Collaboration, Planck 2013 results. XXIII. Isotropy and statistics of the CMB, arXiv:1303.5083 and references therein.

[38] L. Gonzalez-Mestres, Space, Time and Superluminal Particles, arXiv:physics/9702026

[39] L. Gonzalez-Mestres, Spinorial space-time and privileged space direction (I), mp_arc 13-75, and references therein.

[40] L. Gonzalez-Mestres, Spinorial space-time and Friedmann-like equations (I), mp_arc 13-80, and references therein.

[41] L. Gonzalez-Mestres, Pre-Big Bang, space-time structure, asymptotic Universe, these Proceedings.

[42] L. Gonzalez-Mestres, Planck data, spinorial space-time and asymptotic Universe, mp_arc 1333, and references therein.

[43] L. Gonzalez-Mestres, Deformed Lorentz Symmetry and High-Energy Astrophysics (I), ICRC 1999 Evening Workshop Session talk, arXiv:physics/0003080, and references therein.

[44] The OPERA collaboration, Measurement of the neutrino velocity with the OPERA detector in the CNGS beam, arXiv:1109.4897v1

[45] L. Gonzalez-Mestres, Comments on the recent result of the "Measurement of the neutrino velocity with the OPERA detector in the CNGS beam", arXiv:1109.6308

[46] L. Gonzalez-Mestres, Astrophysical consequences of the OPERA superluminal neutrino, arXiv: 1109.6630

[47] L. Gonzalez-Mestres, Lorentz Symmetry Violation and Superluminal Particles at Future Colliders EPS-HEP97, Jerusalem, August 1997, arXiv:physics/9708028, and references therein.

[48] L. Gonzalez-Mestres, Preon models, relativity, quantum mechanics and cosmology (I), arXiv:0908.4070

[49] L. Gonzalez-Mestres, Physics Opportunities Above the Greisen-Zatsepin-Kuzmin Cutoff: Lorentz Symmetry Violation at the Planck Scale, Workshop on "Observing Giant Cosmic Ray Air Showers for > 10E20 eV Particles from Space", University of Maryland, November 1997, AIP Conf. Proc. 433, 148 (1998), arXiv:physics/9712047

[50] ACME (Advanced Cold Molecule Electron EDM) Collaboration, Order of Magnitude Smaller Limit on the Electric Dipole Moment of the Electron, Science 343 (6168) (2014), 269, arXiv: 1310.7534

[51] The Muon (g-2) Collaboration, G.W. Bennett et al., An Improved Limit on the Muon Electric Dipole Moment, Phys.Rev. D 80 (2009), 052008, arXiv: 0811.1207

[52] L. Gonzalez-Mestres, High-Energy Nuclear Physics with Lorentz Symmetry Violation, EPSHEP97 Conference, Jerusalem August 1997, arXiv: nucl-th/9708028

[53] L. Gonzalez-Mestres, Lorentz Symmetry Violation, Vacuum and Superluminal Particles International Conference on Relativistic Physics and some of its Applications, Athens, June 1997, in Open Questions in Relativistic Physics, Ed. F. Selleri, Apeiron 1998, arXiv:physics/9709006

[54] A. De Rújula, R.C. Giles and R.L. Jaffe, Unconfined quarks and gluons, Pys.Rev. D17 (1978), 285. 